\title{
BMJ Open Variations in childbirth interventions in high-income countries: protocol for a multinational cross-sectional study
}

Anna Seijmonsbergen-Schermers, ${ }^{1}$ Ank de Jonge, ${ }^{2}$ Thomas van den Akker, ${ }^{3}$ Katrien Beeckman, ${ }^{4}$ Annick Bogaerts, ${ }^{5,6,7}$ Monalisa Barros, ${ }^{8}$ Patricia Janssen, ${ }^{9}$ Lorena Binfa, ${ }^{10}$ Eva Rydahl, ${ }^{11}$ Lucy Frith, ${ }^{12}$ Mechthild Gross, ${ }^{13}$ Berglind Hálfdánsdóttir, ${ }^{14}$ Deirdre Daly, ${ }^{15}$ Jean Calleja-Agius, ${ }^{16}$ Patricia Gillen, ${ }^{17}$ Anne Britt Vika Nilsen, ${ }^{18}$ Eugene Declercq ${ }^{19}$

To cite: SeijmonsbergenSchermers A, de Jonge A, van den Akker T, et al. Variations in childbirth interventions in high-income countries: protocol for a multinational crosssectional study. BMJ Open 2018;8:e017993. doi:10.1136/ bmjopen-2017-017993

- Prepublication history for this paper is available online. To view these files, please visit the journal online (http://dx.doi. org/10.1136/bmjopen-2017017993).

Received 2 June 2017 Revised 21 November 2017 Accepted 22 November 2017

CrossMark

For numbered affiliations see end of article.

Correspondence to Anna Seijmonsbergen-

Schermers;

a.seijmonsbergen@vumc.nl

\section{ABSTRACT}

Introduction There are growing concerns about the increase in rates of commonly used childbirth interventions. When indicated, childbirth interventions are crucial for preventing maternal and perinatal morbidity and mortality, but their routine use in healthy women and children leads to avoidable maternal and neonatal harm. Establishing ideal rates of interventions can be challenging. This study aims to describe the range of variations in the use of commonly used childbirth interventions in high-income countries around the world, and in outcomes in nulliparous and multiparous women.

Methods and analysis This multinational crosssectional study will use data from births in 2013 with national population data or representative samples of the population of pregnant women in high-income countries. Data from women who gave birth to a single child from 37 weeks gestation onwards will be included and the results will be presented for nulliparous and multiparous women separately. Anonymised individual level data will be analysed. Primary outcomes are rates of commonly used childbirth interventions, including induction and/or augmentation of labour, intrapartum antibiotics, epidural and pharmacological pain relief, episiotomy in vaginal births, instrument-assisted birth (vacuum or forceps), caesarean section and use of oxytocin postpartum. Secondary outcomes are maternal and perinatal mortality, Apgar score below 7 at 5 min, postpartum haemorrhage and obstetric anal sphincter injury. Univariable and multivariable logistic regression analyses will be conducted to investigate variations among countries, adjusted for maternal age, body mass index, gestational weight gain, ethnic background, socioeconomic status and infant birth weight. The overall mean rates will be considered as a reference category, weighted for the size of the study population per country.

Ethics and dissemination The Medical Ethics Review Committee of VU University Medical Center Amsterdam confirmed that an official approval of this study was not required. Results will be disseminated at national and international conferences and published in peer-reviewed journals.
Strengths and limitations of this study

- The use of crude data at the level of individual women.

- The use of strict definitions of variables.

- Multivariable analyses to make more valid comparisons between countries.

- Missing variables in multivariable analyses.

Different quality standards for data across countries.

\section{BACKGROUND}

There are growing concerns about the increase in rates of commonly used childbirth interventions. When indicated, childbirth interventions are crucial for preventing maternal and perinatal morbidity and mortality, and medical technology has led to improved maternal and perinatal outcomes in the last decades ${ }^{1}$ However, the routine use of these interventions in healthy low-risk women and children leads to avoidable maternal and neonatal harm and rising healthcare costs. $^{2-7}$ The rate of childbirth interventions varies both geographically and over different time periods, ${ }^{3}{ }^{8-10}$ even within groups of women with identical risk profiles, and has risen for some interventions and declined for others. ${ }^{8}$ 10-13 In low-income countries, a very low caesarean section (CS) rate has been reported with a high risk of maternal mortality. Due to inadequate access to highquality intrapartum care in these countries, most stillbirths in these countries are preventable. ${ }^{14} \mathrm{CS}$ and labour induction rates have shown a steady increase since the 1970s in high-income countries ${ }^{810121516}$ However, CS rates higher than $10 \%$ are not associated with lower maternal and perinatal mortality. ${ }^{17} 18 \mathrm{In}$ contrast to the increase of CS rates, the episiotomy rate has declined in many countries 
since the 1980s. ${ }^{819}{ }^{20}$ WHO has stated that episiotomy rates should be no higher than $10 \% .^{21}$

While establishing ideal rates of childbirth interventions is a challenge, ${ }^{22} 23$ high rates of interventions should be avoided, especially among low-risk women, because of the risk of adverse effects. CSs are associated with adverse perinatal outcomes, ${ }^{24}$ postoperative complications ${ }^{25} 26$ and avoidable maternal and fetal risks in subsequent pregnancies. ${ }^{27}$ Therefore, CSs should only be performed for strict medical and obstetric indications. Other interventions, such as induction of labour, augmentation of labour, epidural anaesthesia, instrument-assisted birth and episiotomy, if performed without medical indication, can lead to avoidable adverse maternal and perinatal outcomes. ${ }^{28-31}$

Several studies have reported international variations in CS rates. ${ }^{8} 1517$ 32-34 Positive correlations with CS rates have been shown for gross domestic product per capita, proportion of the population living in urban areas, number of doctors and for the presence of a skilled birth. An increased percentage of childbirth interventions in private hospitals compared with public hospitals has also been described. ${ }^{15} 1732$

Few studies have focused on variations in childbirth interventions other than CS or rates of several childbirth interventions together. In 1990, Notzon described sharp differences in rates of CSs in 21 countries and rates of instrument-assisted births in 14 countries. ${ }^{9}$ In 1993 , Stephenson et al described a 3-fold difference in CSs and a 10-fold difference in instrument-assisted vaginal births in 12 countries. ${ }^{35}$ The European Perinatal Health Report confirmed major variation in rates of interventions such as CSs, instrument-assisted births, induction of labour, episiotomy and births without childbirth interventions in 2004 and 2010 in Europe. ${ }^{8}$ For example, a secondary analysis of these data showed that national episiotomy rates in all vaginal births ranged from $4.9 \%$ in Denmark to $75.0 \%$ in Cyprus. ${ }^{36}$ Festin et $a P^{37}$ found significant variation in the practice of active management of the third stage of labour in 10 countries. ${ }^{37}$ Patterns of associations between childbirth interventions, such as CSs and the use of instruments, and epidurals and intrapartum oxytocin use, have been shown. ${ }^{5838} 39$

In addition to the variation in childbirth interventions, intercountry variations in maternal and perinatal outcomes also exist. ${ }^{80}$ For example, the proportion of women with obstetric anal sphincter injury (OASI) ranged from $0.1 \%$ in Poland and Romania and $0.3 \%$ in Slovenia to over $4 \%$ in Denmark and Iceland. ${ }^{36}$ While the maternal mortality rate in most countries in Europe has not declined significantly over the past decades, in some countries, including the USA, this rate has increased. ${ }^{841}$ Perinatal mortality rates have declined in many countries worldwide and wide variations persist; however, these figures might be influenced by registration differences and under-reporting in some countries. ${ }^{842}{ }^{43}$ Since differences in registration between countries occur, data must be interpreted with this in mind and figures of intervention rates must not be analysed without describing maternal and perinatal outcomes.

Because of the wide variations in rates of childbirth interventions and maternal and perinatal outcomes that exist between countries, it is important that childbirth interventions are analysed comprehensively, so that rates of several interventions can be compared and patterns can be better understood. This is the first study comparing rates of several interventions with the use of clear definitions of the interventions and outcomes to facilitate meaningful comparisons. This study aims to describe the range of variations in commonly used childbirth interventions in nulliparous and multiparous women in high-income countries around the world, and outcomes, by comparing these rates among multiple countries.

\section{METHODS AND ANALYSIS}

\section{Research design and setting}

This multinational cross-sectional study uses existing data from childbearing women in 2013. The year 2013 was chosen to ensure that required data were available for all participating countries, and were as recent as possible. Data will be analysed in the Netherlands, during the autumn of 2017 and analyses will continue throughout 2018. High-income countries were selected on the basis of their representation in the COST Action IS1405 'BIRTH' (European Cooperation in Science and Technology), ${ }^{44}$ and whether the necessary data for this study were available. Low-income countries are not included because of the difficulties in comparing them with high-income countries due to differences in healthcare services and access to care. ${ }^{45}$ Countries that were not in the COST Action were also invited to reach more global coverage, and so the use of interventions among countries with diverse maternity care systems could be compared. Participating countries are Belgium, Canada, Chile, Denmark, England, Germany, Iceland, Ireland, Italy, Malta, the Netherlands, Northern Ireland, Norway and the USA. The reporting of this study will adhere to the Strengthening the Reporting of Observational Studies in Epidemiology guidelines.

\section{Participants}

Data from all women in participating countries, or from a representative sample of the population, who gave birth to a single child from 37 weeks gestation onwards in 2013 are eligible for inclusion. For countries that cannot provide data from all women who gave birth in 2013, a representative sample of the population will be included. Which proportion of the total population it concerns, will be clearly described. Sample size calculation was not performed, because all available and eligible data from the participating countries for the year 2013 were included. Countries for which no data on parity, gestational age at birth and singleton or multiple gestation were available, were excluded. 


\section{Patient involvement}

Informed consent was not sought and women were not involved in the design of this study, because only anonymised national data or data from a representative sample of the population from 2013 will be used, which in most countries was routinely collected or collected after informed consent.

\section{Outcome measures}

\section{Primary outcomes}

The following variables are defined as primary outcomes: induction of labour (none; cervical ripening; artificial rupture of membranes; oxytocin; other method; unspecified), augmentation of labour, intrapartum use of oxytocin, artificial rupture of membranes, intrapartum antibiotics, epidural/spinal anaesthesia for labour (CS excluded), other pharmacological pain relief (none; systemic (non-)opioid analgesia; inhaled nitrous oxide; other; unspecified), episiotomy in vaginal births (none; mediolateral; midline; unspecified), spontaneous vaginal birth, instrument-assisted birth (International Classification of Diseases (ICD) code 081) (none; vacuum extraction; forceps delivery; unspecified), CS (ICD code 082) (none; planned; emergency; unspecified), active management of third stage of labour and use of oxytocin within 2 hours postpartum.

\section{Secondary outcomes}

Secondary outcomes are perinatal mortality, Apgar score below 7 at $5 \mathrm{~min}$, maternal mortality, postpartum haemorrhages $\geq 1000 \mathrm{~mL}$ (ICD code 072) and OASI (ICD code 070.2 and 070.3$)$.

\section{Definitions}

Clear operational definitions of the interventions and childbirth outcome measures will be used in order to allow valid international comparisons. Definitions of variables will vary among the countries. To ensure optimal uniformity, detailed instructions for the definitions of the variables will be given to the investigators.

\section{Spontaneous onset of labour}

Spontaneous onset of labour means spontaneous onset of regular uterine contractions that was not preceded by cervical ripening or the use of any other method of inducing uterine contractions.

\section{Induction of labour}

For induction of labour, more than one method can be used. Induction is defined as a technique to stimulate uterine contractions artificially prior to spontaneous contractions and includes administration of oxytocin to stimulate uterus contractions after spontaneous ruptured membranes in the absence of uterine contractions, or administration of oxytocin after cervical ripening and/ or artificial rupture of membranes. ${ }^{46}$ Cervical ripening is defined as an application of cervical ripening agents (such as prostaglandins) or mechanical interventions (such as insertion of catheters) and is also included in the definition of induction of labour.

\section{Augmentation of labour}

Augmentation of labour is the administration of oxytocin to stimulate uterus contractions after spontaneous onset of labour. ${ }^{47}$

\section{Artificial rupture of membranes}

Artificial rupture of membranes means breaking the amniotic fluid sac artificially, regardless of the moment (before or during labour) or indication (eg, for induction of labour).

\section{Epidural anaesthesia}

Epidural/spinal/neuroaxial anaesthesia refers to its use for pain management during labour contractions (spontaneous or induced). In case of a planned CS, epidural anaesthesia is considered not applicable and will be defined as no epidural anaesthesia.

\section{Episiotomy in vaginal births}

An episiotomy is the use of a deliberate incision of the perineum to accelerate vaginal birth. ${ }^{48}$ Episiotomy will be described for women with vaginal births only.

\section{Caesarean section}

Planned CS is defined as a CS that was planned before onset of labour and was performed electively before or after spontaneous onset of labour. Emergency CS is defined as a CS that was not planned prior to the onset of labour.

\section{Active management of the third stage of labour and oxytocin in the postpartum period}

Active management of the third stage of labour is defined as a combination of administration of an uterotonic agent after birth, early cord clamping and controlled cord traction. For use of oxytocin in the postpartum period, oxytocin can be administered intravenously or intramuscularly within 2 hours postpartum, including oxytocin use for induction or augmentation of labour which is continued after birth.

\section{Mortality}

Perinatal and maternal mortality are defined as mortality within 7 days postpartum.

\section{Obstetric anal sphincter injury}

OASI is a tear that extends to the external anal sphincter or when the rectal mucosa is torn.

\section{Countries and data source characteristics in 2013}

The following data will be collected to provide an overview of the characteristics of the countries represented in this study: total number of inhabitants, number of births in the country in 2013, proportion of births in the provided dataset, Gross National Income per capita in 2013 (as specified by the World Bank ${ }^{45}$ ), source of the data (civil registration; medical birth register or child health system; 
hospital discharge system; perinatal survey; confidential enquiry; other routine surveys; linked data source) and the organisation responsible for providing the data.

\section{Independent variables}

Independent variables are parity (nulliparous; multiparous), maternal age at the time of giving birth $(<20$; 20-24; 25-29; 30-34; 35-39; $\geq 40$ years), maternal body mass index (BMI) $(<18.5 ; 18.5-24.9 ; 25.0-29.9 ; 30.0-$ $\left.34.9 ; 35.0-39.9 ; \geq 40.0 \mathrm{~kg} / \mathrm{m}^{2}\right)$, gestational weight gain (mean and IQR, in grams), ethnic background, socioeconomic status (high; medium; low), gestational age at birth ( $37 ; 38 ; 39 ; 40 ; 41 ; \geq 42$ weeks), birth weight $(<2500$; 2500-3499; 3500-4499; $\geq 4500 \mathrm{~g}$ ), place of birth (hospital; birth centre; home; other) and care provider responsible at onset of labour and at birth (midwife; obstetrician/gynaecologist; nurse midwife; general practitioner; other). Categories for ethnic background will depend on national classifications and will therefore vary between countries. The definition of ethnicity that is used in each country will be used to describe ethnicity in the descriptive analyses. When data from all countries are collected, an attempt will be made to provide a uniform definition (if possible) to enable adjustment for ethnicity. It may not be possible for most of the countries to collect data on whether a woman is a refugee or a migrant (as definitions of 'migrant' vary across the countries). The variable socioeconomic status will be categorised as high (75th percentile), medium (25th-75th percentile) and low (25th percentile) and the definition will also depend on national classifications. Some countries are not able to provide information on socioeconomic status and will therefore provide another variable that relates to socioeconomic status, such as education. How socioeconomic status is defined, will be clearly described for each country. An attempt will be made to provide a uniform definition (if possible) to enable adjustment for socioeconomic status. Place of birth will be categorised as in a hospital (a hospital where a CS can be performed), birth centre (a centre or hospital where a CS cannot be performed), home and other.

\section{Analysis}

The results will be analysed and presented by country and will be stratified by parity. The results will be reported in absolute numbers and percentages. Univariable analyses will be performed to report on variations in rates of interventions and multivariable logistic regression analyses will be conducted to investigate variations among countries adjusted for maternal age, maternal BMI, gestational weight gain, ethnic background, socioeconomic status and infant birth weight. Because stratification by parity will not be used for outcomes with only a few cases, for these outcomes adjustments for parity will be added. ORs and 99\% CIs will be used to compare the probability of interventions and the risk for maternal and perinatal outcomes compared with the weighted mean rate of the complete dataset, to ensure that all countries contribute equally to the analyses. For the multivariable logistic regression analyses, outcome variables will be dichotomised and dummy variables will be created for potential confounders. To give a better understanding of the relationships between interventions, the results of interventions will be linked (such as emergency CS and instrument-assisted births) and illustrated in box plots with adjusted ORs and 99\% CIs. Statistical analyses will be performed using STATA V.14 (StataCorp, College Station, Texas, USA).

\section{Missing data}

The amount and type of missing data will be reported for each variable by country. If a country's dataset has no information about a specific variable or has data regarding a dependent variable with $>10 \%$ missing, the country's data will be excluded from the analysis for that specific variable. If a an independent variable included in the multivariable analyses is available, but there is $>5 \%$ missing, data will be imputed using multiple imputation.

\section{Ethical and data protection issues}

Investigators will be asked to describe their national ethical standards and data protection laws. They will also be asked to report the proportion of cases for which data are missing in the dataset. Countries for which no anonymised crude data at the level of individual women can be provided, will participate with aggregated data and these data will be used in the descriptive analyses. Finally, investigators will be asked to provide information on the standard quality procedures of the data source and the provided dataset, how missing data are dealt with and contradictions, and how checks are done to ensure that all eligible births are recorded. If the conditions for participation of the country are met, a data access agreement will be signed between the investigator's institution and the VU University Medical Center.

\section{ETHICS AND DISSEMINATION}

The Medical Ethics Review Committee of VU University Medical Center Amsterdam confirmed that official approval of this study was not required, because it concerns a secondary analysis of existing data (reference 2016.317). This multinational cross-sectional study with data from high-income countries will describe variations in childbirth interventions and maternal and perinatal outcomes. A comparison of intervention rates and rates of childbirth outcomes between countries will identify opportunities to improve maternal and perinatal outcomes. Results will be disseminated at national and international conferences and published in peer-reviewed journals.

\section{DISCUSSION}

For some interventions, there is lack of consensus and ongoing debate about indications for their use and 
whether an intervention is necessary, for example, planned CS for breech presentations, or after a previous $\mathrm{CS},{ }^{8}$ or the use of episiotomy for prevention of major perineal tears in nulliparous and multiparous women. ${ }^{49}$ It is important to avoid unnecessary interventions in childbearing women and at the same time ensure that those interventions that are necessary take place.

This study only aims to describe variation in intervention rates, related to a number of maternal and birth characteristics. The design of this study is inappropriate for making causal associations. Therefore, the results need to be interpreted with caution. Indications for the use of childbirth interventions are rarely reported clearly in national data. Although multivariable analyses are conducted to adjust for characteristics, the indications for the use of interventions cannot be included in this study. Also other potential maternal confounders such as pre-existing medical condition and previous history cannot be included. This limits interpretation of the results, and reveals at the same time that auditing indications of childbirth interventions is important to ensure better comparisons between countries in future studies.

A major strength of this study is the use of crude data at the level of individual women and the use of strict definitions. This will allow us to conduct multivariable analyses and make valid comparisons between countries. As described before, the literature shows patterns of associations between commonly used childbirth interventions. Our data will permit exploration of these associations between interventions, which will give a better understanding of the patterns of intervention use and the impact of variations on mother and child.

Studying variations in a large number of interventions across countries will inform the debate about optimal rates and the results will contribute to the improvement of the quality of care. In subsequent studies, factors influencing major variations in intervention rates can be examined and addressed.

\section{Author affiliations}

${ }^{1}$ Department of Midwifery Science, AVAG, Amsterdam Public Health Research Institute, VU University Medical Center, Amsterdam, The Netherlands

${ }^{2}$ Department of Midwifery Science, VU University Medical Center, Amsterdam, The Netherlands

${ }^{3}$ Department of Obstetrics, Leiden University Medical Center, Leiden, The Netherlands

${ }^{4}$ Nursing and Midwifery Research Unit, Faculty of Medicine and Pharmacy, Vrije Universiteit Brussel, Brussel, Belgium

${ }^{5}$ Department of Development and Regeneration KU Leuven, University of Leuven,

Leuven, Belgium

${ }^{6}$ Faculty of Medicine and Health Sciences, Centre for Research and Innovation in Care (CRIC), University of Antwerp, Antwerp, Belgium

${ }^{7}$ Faculty of Health and Social Work, Research Unit Healthy Living, Uc LeuvenLimburg, Leuven, Belgium

${ }^{8}$ Universidade Estadual do Sudoeste da Bahia, Vitória da Conquista, Brazil

${ }^{9}$ University of British Columbia, Vancouver, Canada

${ }^{10}$ Department of Women's and New Born Health Promotion-School of Midwifery

Faculty of Medicine, University of Chile, Santiago, Chile

${ }^{11}$ Department of Midwifery, Metropolitan University College, Copenhagen, Denmark
${ }^{12}$ Department of Health Services Research, The University of Liverpool, Liverpool, UK ${ }^{13}$ Midwifery Research and Education Unit, Department of Obstetrics, Gynaecology and Reproductive Medicine, Hannover Medical School, Hannover, Germany

${ }^{14}$ Midwifery Programme, Faculty of Nursing, School of Health Sciences, University of Iceland, Reykjavík, Iceland

${ }^{15}$ School of Nursing and Midwifery, Trinity College Dublin, Dublin, Ireland

${ }^{16}$ Department of Anatomy, Faculty of Medicine and Surgery, University of Malta, Tal-Qroqq, Malta

${ }^{17}$ Institute of Nursing and Health Research, Ulster University, Jordanstown, UK

${ }^{18}$ Western Norway University of Applied Sciences (HVL), Bergen, Norway

${ }^{19}$ Boston University School of Public Health, Boston, Massachusetts, USA

Acknowledgements We acknowledge the contribution of $\mathrm{F}$ Schellevis for his input and advice into the design of this study. We also acknowledge the contribution of: M Willems from Statistics Belgium, Federal Public Services and the 'Gemeenschappelijke Gemeenschapscommissie voor de geboorten en overlijdens in het Brusselse Gewest', the 'Vlaamse Gemeenschap' and 'the Federatie WalloniëBrussel' for providing the data for Belgium; DataSus and Fiocruz for providing the data for Brazil; The Canadian Institute for Health Information for providing the data for Canada; The Ministry of Health Chile (2013), Department of Statistics and Health Information, Data Base of Birth and Hospital Discharges 2013 for providing the data for Chile; The Statistics Denmark for providing the data for Denmark; The Health and Social care information centre - Hospital Episode Statistics NHS Maternity Statistics - England, 2013-14 for providing the data for England; Geschaeftsstelle Qualitaetssicherung Hessen (Institute for Quality Assurance Hesse) for providing the data for Germany; The Icelandic National Birth Registry for providing the data for Iceland; The Healthcare Pricing Office for providing the data for Ireland; The Department of Health Information and Research for providing data from the National Obstetric Information System (NOIS) for Malta; The Netherlands Perinatal Registry for providing the data for the Netherlands; The Northern Ireland Statistics \& Research Agency NISRA for providing the data for Northern Ireland; The Medical Birth Registry of Norway and Statistics Norway for providing the data for Norway; The National Vital Statistics System for providing the data for the United States. This article is based upon work from COST Action IS1405 'BIRTH': "Building Intrapartum Research Through Health - an interdisciplinary whole system approach to understanding and contextualising physiological labour and birth" (http://www. cost.eu/COST_Actions/isch/IS1405), supported by COST (European Cooperation in Science and Technology).

Contributors This study protocol was developed by AS-S and AJ, in collaboration with TA at the Department of Midwifery Science, AVAG and the EMGO Institute of Health and Care Research in Amsterdam, The Netherlands. AS-S wrote the paper and will lead the data analyses, in collaboration with $A J . A J, T A, K B, A B, M B, P J, L B$, $E R, L F, M G, B H, D D, P G, J C-A, A B V N$ and ED critically revised earlier drafts of this protocol, have approved the final version of this protocol and will review the results and drafts of the manuscript. KB, AB, PJ, LB, ER, LF, MG, BH, DD, PG, JC-A, ABVN and $E D$ will contribute to this study by preparing and delivering data of their country and/or check the analyses of the data of their country.

Funding The study was developed during a meeting with COST-members (European Cooperation in Science and Technology). These meetings are funded by the COST Action IS1405 'BIRTH' (European Cooperation in Science and Technology). There is no other external funding for this study.

Competing interests None declared.

Ethics approval Medical Ethics Review Committee of the VU University Medical Center Amsterdam.

Provenance and peer review Not commissioned; externally peer reviewed.

Data sharing statement Patient-level data and full dataset are available, but restrictions apply to the availability of these data which were used under license for the current study, and so are not publicly available. Consent was not obtained but the presented data are anonymised and risk of identification is low.

Open Access This is an Open Access article distributed in accordance with the Creative Commons Attribution Non Commercial (CC BY-NC 4.0) license, which permits others to distribute, remix, adapt, build upon this work non-commercially, and license their derivative works on different terms, provided the original work is properly cited and the use is non-commercial. See: http://creativecommons.org/ licenses/by-nc/4.0/

(C) Article author(s) (or their employer(s) unless otherwise stated in the text of the article) 2018. All rights reserved. No commercial use is permitted unless otherwise expressly granted. 


\section{REFERENCES}

1. Woods R. Long-term trends in fetal mortality: implications for developing countries. Bull World Health Organ 2008;86:460-6.

2. Kilsztajn S, Carmo MS, Machado LC, et al. Caesarean sections and maternal mortality in Sao Paulo. Eur J Obstet Gynecol Reprod Biol 2007;132:64-9.

3. Lumbiganon P, Laopaiboon M, Gülmezoglu AM, et al. Method of delivery and pregnancy outcomes in Asia: the WHO global survey on maternal and perinatal health 2007-08. Lancet 2010;375:490-9.

4. Renfrew MJ, McFadden A, Bastos MH, et al. Midwifery and quality care: findings from a new evidence-informed framework for maternal and newborn care. Lancet 2014;384:1129-45.

5. Tracy SK, Sullivan E, Wang YA, et al. Birth outcomes associated with interventions in labour amongst low risk women: a population-based study. Women Birth 2007;20:41-8.

6. Villar J, Valladares E, Wojdyla D, et al. Caesarean delivery rates and pregnancy outcomes: the 2005 WHO global survey on maternal and perinatal health in Latin America. Lancet 2006;367:1819-29.

7. Miller S, Abalos E, Chamillard M, et al. Beyond too little, too late and too much, too soon: a pathway towards evidence-based, respectful maternity care worldwide. Lancet 2016;388:2176-92.

8. EURO-PERISTAT Project with SCPE and EUROCAT. European Perinatal Health Report. Health and care of pregnant women and babies in Europe in 2010. 2013 www.europeristat.com (accessed 29 May 2017).

9. Notzon FC. International differences in the use of obstetric interventions. JAMA 1990;263:3286-91.

10. Vogel JP, Gülmezoglu AM, Hofmeyr GJ, et al. Global perspectives on elective induction of labor. Clin Obstet Gynecol 2014;57:331-42.

11. Dublin S, Johnson KE, Walker RL, et al. Trends in elective labor induction for six United States health plans, 2001-2007. J Womens Health 2014;23:904-11.

12. Martin JA, Hamilton BE, Osterman MJ. Births in the United States, 2013. NCHS Data Brief 2014:1-8.

13. Osterman MJ, Martin JA. Recent declines in induction of labor by gestational age. NCHS Data Brief 2014;155:1-8.

14. The Lancet Ending Preventable Stillbirths study group. Ending preventable stillbirths [Internet]. 2016 http://www.thelancet.com/ $\mathrm{pb} /$ assets/raw/Lancet/stories/series/stillbirths2016-exec-summ.pdf (accessed 16 Sep 2017).

15. Cavallaro FL, Cresswell JA, França GV, et al. Trends in caesarean delivery by country and wealth quintile: cross-sectional surveys in southern Asia and sub-Saharan Africa. Bull World Health Organ 2013;91:914-22.

16. Patterson JA, Roberts CL, Ford JB, et al. Trends and outcomes of induction of labour among nullipara at term. Aust N Z J Obstet Gynaecol 2011;51:510-7.

17. Betrán AP, Merialdi M, Lauer JA, et al. Rates of caesarean section: analysis of global, regional and national estimates. Paediatr Perinat Epidemiol 2007:21:98-113.

18. Betran AP, Torloni MR, Zhang JJ, et al. WHO statement on caesarean section rates. BJOG 2016;123:667-70.

19. Weber AM, Meyn L. Episiotomy use in the United States, 1979-1997. Obstet Gynecol 2002;100:1177-82.

20. Suzuki S, Satomi M. Episiotomy in the United States: has anything changed? Am J Obstet Gynecol 2010;202:e5-8.

21. World Health Organization. Care in normal birth: a practical guide. 1996.

22. Gibbons L, Belizán JM, Lauer JA. 2010. The global numbers and costs of additionally needed and unnecessary caesarean sections performed per year: overuse as a barrier to universal coverage. world health report. Geneva: WHO. Background Paper 30.

23. Betran AP, Torloni MR, Zhang J, et al. What is the optimal rate of caesarean section at population level? A systematic review of ecologic studies. Reprod Health 2015;12:57.

24. Annibale DJ, Hulsey TC, Wagner CL, et al. Comparative neonatal morbidity of abdominal and vaginal deliveries after uncomplicated pregnancies. Arch Pediatr Adolesc Med 1995;149:862-7.

25. Shorten A. Maternal and neonatal effects of caesarean section. BMJ 2007;335:1003-4.
26. van Ham MA, van Dongen PW, Mulder J. Maternal consequences of caesarean section. A retrospective study of intra-operative and postoperative maternal complications of caesarean section during a 10-year period. Eur J Obstet Gynecol Reprod Biol 1997;74:1-6.

27. Taylor LK, Simpson JM, Roberts CL, et al. Risk of complications in a second pregnancy following caesarean section in the first pregnancy: a population-based study. Med J Aust 2005;183:515-9.

28. Anim-Somuah M, Smyth RM, Jones L. Epidural versus nonepidural or no analgesia in labour. Cochrane Database Syst Rev 2011;12:CD000331.

29. Bonsack CF, Lathrop A, Blackburn M. Induction of labor: update and review. J Midwifery Womens Health 2014;59:606-15.

30. Doumouchtsis SK, Arulkumaran S. Head trauma after instrumental births. Clin Perinatol 2008;35:69-83.

31. Liu S, Heaman M, Joseph KS, et al. Risk of maternal postpartum readmission associated with mode of delivery. Obstet Gynecol 2005;105:836-42.

32. Belizán JM, Althabe F, Barros FC, et al. Rates and implications of caesarean sections in Latin America: ecological study. BMJ 1999;319:1397-400.

33. Notzon FC, Placek PJ, Taffel SM. Comparisons of national cesareansection rates. $N$ Engl J Med 1987;316:386-9.

34. Zizza A, Tinelli A, Malvasi A, et al. Caesarean section in the world: a new ecological approach. J Prev Med Hyg 2011;52:161-73.

35. Stephenson PA, Bakoula C, Hemminki E, et al. Patterns of use of obstetrical interventions in 12 countries. Paediatr Perinat Epidemiol 1993;7:45-54.

36. Blondel B, Alexander S, Bjarnadóttir RI, et al. Variations in rates of severe perineal tears and episiotomies in 20 European countries: a study based on routine national data in Euro-Peristat Project. Acta Obstet Gynecol Scand 2016;95:746-54.

37. Festin MR, Lumbiganon P, Tolosa JE, et al. International survey on variations in practice of the management of the third stage of labour. Bull World Health Organ 2003;81:286-91.

38. Rossignol M, Chaillet N, Boughrassa F, et al. Interrelations between four antepartum obstetric interventions and cesarean delivery in women at low risk: a systematic review and modeling of the cascade of interventions. Birth 2014;41:70-8.

39. Robson SJ, de Costa CM. Thirty years of the world health organization's target caesarean section rate: time to move on. Med $J$ Aust 2017;206:181-5.

40. Kassebaum NJ, Bertozzi-Villa A, Coggeshall MS, et al. Global, regional, and national levels and causes of maternal mortality during 1990-2013: a systematic analysis for the global burden of disease study 2013. Lancet 2014;384:980-1004.

41. MacDorman MF, Declercq E, Cabral H, et al. Recent increases in the U.S. maternal mortality rate: disentangling trends from measurement issues. Obstet Gynecol 2016;128:447-55.

42. Rajaratnam JK, Marcus JR, Flaxman AD, et al. Neonatal, postneonatal, childhood, and under-5 mortality for 187 countries, 1970-2010: a systematic analysis of progress towards Millennium development goal 4. Lancet 2010;375:1988-2008.

43. Blencowe H, Cousens S, Jassir FB, et al. National, regional, and worldwide estimates of stillbirth rates in 2015, with trends from 2000 : a systematic analysis. Lancet Glob Health 2016;4:e98-e108.

44. EU Birth Research Project. A COST Action website detailing research into birth practices. Information about the EU Birth Research Project. https://eubirthresearch.eu/ (accessed 10 Dec 2016).

45. The World Bank Group. World bank open data. Free and open acces to global development data [Internet]. 2017 http://data.worldbank. org/ (accessed 29 May 2017).

46. WHO Guidelines Approved by the Guidelines Review Committee. WHO recommendations for induction of labour. Geneva: World Health Organization, 2011.

47. World Health Organization. WHO recommendations for augmentation of labour. Geneva: World Health Organization, 2014.

48. Jiang H, Qian X, Carroli G, et al. Selective versus routine use of episiotomy for vaginal birth. Cochrane Database Syst Rev 2017;2:CD000081.

49. Seijmonsbergen-Schermers AE, Geerts CC, Prins M, et al. The use of episiotomy in a low-risk population in the Netherlands: a secondary analysis. Birth 2013;40:247-55. 
Correction: Variations in childbirth interventions in highincome countries: protocol for a multinational cross-sectional study

Seijmonsbergen-Schermers A, de Jonge A, van den Akker $\mathrm{T}$, et al. Variations in childbirth interventions in high-income countries: protocol for a multinational crosssectional study. BMJ Open 2018;8:e017993. doi: 10.1136/bmjopen-2017-017993.

The author order is incorrect in the article. The correct author order should be:

Anna Seijmonsbergen-Schermers, Thomas van den Akker, Katrien Beeckman, Annick Bogaerts, Monalisa Barros, Patricia Janssen, Lorena Binfa, Eva Rydahl, Lucy Frith, Mechthild M Gross, Berglind Hálfdánsdóttir, Deirdre Daly, Jean Calleja-Agius, Patricia Gillen, Anne Britt Vika Nilsen, Eugene Declercq, Ank de Jonge.

Open Access This is an Open Access article distributed in accordance with the Creative Commons Attribution Non Commercial (CC BY-NC 4.0) license, which permits others to distribute, remix, adapt, build upon this work non-commercially, and license their derivative works on different terms, provided the original work is properly cited and the use is non-commercial. See: http://creativecommons.org/licenses/by-nc/4.0/

C Article author(s) (or their employer(s) unless otherwise stated in the text of the article) 2018. All rights reserved. No commercial use is permitted unless otherwise expressly granted.

BMJ Open 2018;8:e017993corr1. doi:10.1136/bmjopen-2017-017993corr1

Check for updates 